\title{
Uncertainty Analysis of Light Water Reactor Fuel Lattices
}

\author{
C. Arenas, ${ }^{1}$ R. Bratton, ${ }^{2}$ F. Reventos, ${ }^{1}$ and K. Ivanov ${ }^{2}$ \\ ${ }^{1}$ Department of Physics and Nuclear Engineering, Technical University of Catalonia, 08034 Barcelona, Spain \\ ${ }^{2}$ Department of Mechanical and Nuclear Engineering, The Pennsylvania State University, University Park, PA 16802, USA
}

Correspondence should be addressed to C. Arenas; carenas@tinet.org

Received 20 August 2012; Accepted 18 November 2012

Academic Editor: Carlo Parisi

Copyright $\odot 2013$ C. Arenas et al. This is an open access article distributed under the Creative Commons Attribution License, which permits unrestricted use, distribution, and reproduction in any medium, provided the original work is properly cited.

\begin{abstract}
The study explored the calculation of uncertainty based on available cross-section covariance data and computational tool on fuel lattice levels, which included pin cell and the fuel assembly models. Uncertainty variations due to temperatures changes and different fuel compositions are the main focus of this analysis. Selected assemblies and unit pin cells were analyzed according to the OECD LWR UAM benchmark specifications. Criticality and uncertainty analysis were performed using TSUNAMI-2D sequence in SCALE 6.1. It was found that uncertainties increase with increasing temperature, while $k_{\text {inf }}$ decreases. This increase in the uncertainty is due to the increase in sensitivity of the largest contributing reaction of uncertainty, namely, the neutron capture reaction ${ }^{238} \mathrm{U}(n, \gamma)$ due to the Doppler broadening. In addition, three types (UOX, MOX, and UOX- $\mathrm{Gd}_{2} \mathrm{O}_{3}$ ) of fuel material compositions were analyzed. A remarkable increase in uncertainty in $k_{\text {inf }}$ was observed for the case of MOX fuel. The increase in uncertainty of $k_{\text {inf }}$ in MOX fuel was nearly twice the corresponding value in UOX fuel. The neutron-nuclide reaction of ${ }^{238} \mathrm{U}$, mainly inelastic scattering $\left(n, n^{\prime}\right)$, contributed the most to the uncertainties in the MOX fuel, shifting the neutron spectrum to higher energy compared to the UOX fuel.
\end{abstract}

\section{Introduction}

The demand for the best estimate calculations in nuclear reactor core modeling and design has increased in recent years. Uncertainty analysis has been highlighted as an important part of the design and safety analysis of modern nuclear reactors. The modeling aspects of uncertainty analysis and sensitivity analysis are to be further developed and validated on scientific grounds in support of their performance. The Organization for Economic Cooperation and Development (OECD)/Nuclear Energy Agency (NEA) initiated the Benchmark for Uncertainty Analysis in Modeling, Design, Operation, and Safety Analysis of Light Water Reactor (OECD LWR UAM benchmark). The general objective of the benchmark is to propagate the uncertainty through complex coupled multiphysics and multiscale simulations. The benchmark is divided into three phases with Phase I highlighting the uncertainty propagation in neutronics calculations, while Phases II and III are focused on uncertainty analysis of reactor core and reactor system, respectively.

In Phase I of the OECD LWR UAM benchmark, the exercises are divided into three parts: cell physics (Exercise I), lattice physics (Exercise II), and core physics (Exercise III) [1] . This paper will discuss Exercises I and II.

\section{Uncertainty Calculations}

In general, uncertainty is calculated based on covariance matrix and weighting factor coefficients [2]:

$$
\Delta^{2}=\alpha[\operatorname{cov} v] \alpha^{T},
$$

where $\alpha$ is the weighting factor matrix, $\alpha^{T}$ is the transpose of the weighting factor matrix, $[\mathrm{cov}]$ is the covariance matrix, and $\Delta^{2}$ is the uncertainty.

In order to obtain the uncertainty of the response of interest, which may be the critical eigenvalue, the reactivity difference between two reactor states, or the ratio of reactions rates, sensitivity coefficients $(S)$ are used as weighting factors to the covariance matrix

$$
\Delta_{k \text { eff }}^{2}=S\left[\operatorname{cov} v S^{T} .\right.
$$


2.1. Covariance Matrix of Nuclear Data. The evaluation of nuclear data induced uncertainty is possible by the use of nuclear cross-section variance and covariance data. By including the uncertainty or covariance information, the analyst can propagate cross-section data uncertainties through sensitivity studies to the final calculated quantities of interest. The covariance data files provide the estimated variance for the individual data as well as any correlation that may exist. In principle, the covariance matrices can be now self-shielded in the same way as the cross-sections, although in practice this is rarely done. The impact of this treatment on the obtained covariance matrices and their dependence on energy group structure needs to be studied. The SCALE 6.1/TSUNAMI-2D [3] sequence uses a completely different approach to address the impact of self-shielding in sensitivity and uncertainty analysis. Rather than modifying the covariance data, the sensitivity coefficients are modified to include the "implicit effects" of perturbations in the group cross-sections caused by perturbations in self-shielding. Implicit effects account for impact of resonance self-shielding on sensitivity coefficients and uncertainty evaluations. This allows the use of unshielded covariance data. Treatment of implicit effects is a standard part of the TSUNAMI-2D analysis and has been shown to be a significant sensitivity component in some cases.

The SCALE 6.1 covariance library data corresponds also to 44-group relative uncertainties assembled from a variety of sources, including evaluations from ENDF/B-VII, ENDF/BVI, JENDL-3.3, and more than 300 approximated uncertainties from a collaborative project performed by Brookhaven National Laboratory (BNL), Los Alamos National Laboratory (LANL), and Oak Ridge National Laboratory (ORNL).

It is assumed that the same relative (rather than absolute) uncertainties can be applied to all cross-section libraries, even if these are not strictly consistent with the nuclear data evaluations. In addition, the assumption that there are no covariance correlations between energy groups is applied [1].

2.2. Sensitivity Coefficients. For light water reactors, two components of sensitivity coefficient are needed. Explicit sensitivity represents the sensitivity of the calculated $k_{\text {inf }}$ to components of the group-wise cross-section data. Implicit sensitivity addresses the impact of resonance self-shielding effect in a system. Together, the explicit and implicit sensitivity are combined to produce the total sensitivity:

$$
S_{\text {Total }}=S_{\text {Explicit }}+S_{\text {Implicit }} \text {. }
$$

2.3. Computational Tools. The SCALE 6.1/TSUNAMI-2D sequence is used to perform the study. First, the ENDF/BVII.0 based 238-group microscopic cross-section data library is processed using BONAMIST and CENTRM/PMC. Next, forward and adjoint calculations are performed using NEWT, a 2D transport solver. Finally, sensitivity coefficients are calculated, and uncertainty data is generated by SAMS using the default covariance data library in 44 groups (44groupcov).

The SCALE 6.1 sensitivity and uncertainty methodology is based on the first-order perturbation theory to calculate response sensitivity coefficients, which are then folded with nuclear data covariances to obtain the response uncertainty.

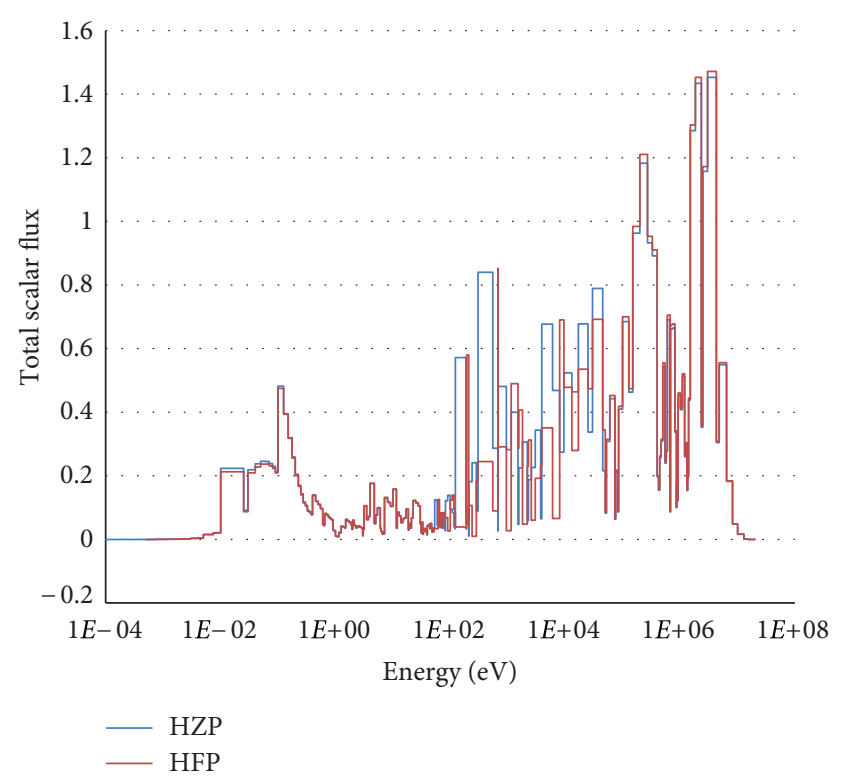

Figure 1: The neutron flux of PWR unit cell at two different operating conditions.

TSUNAMI-2D applies the generalized perturbation theory (GPT) to generate uncertainties associated with the fewgroup assembly homogenized neutron cross-section data [2].

\section{Temperature Sensitivity Study}

The study begins with specification of fuel pin cells of three light water reactor types and two critical experiments provided by the OECD LWR UAM benchmark within Exercise I-1 [4]. This exercise evaluates the multigroup microscopic cross-section uncertainties associated with multigroup crosssection libraries used as an input in lattice physics codes. The details of the specifications are available in [1]. $k_{\text {inf }}$ and propagated cross-section uncertainties obtained for different pin cell test problems are shown in Table 1.

Observations of the results showed that $k_{\text {inf }}$ reduces as the temperature rises. This is due to the effect of Doppler broadening and the reduction in moderation due to the changing moderator density. The uncertainty in $k_{\text {inf }}$ seemed to increase with increasing temperature. The largest contributor to the uncertainty is due to ${ }^{238} \mathrm{U}(n, \gamma)$ reaction. The majority of neutron capture in ${ }^{238} \mathrm{U}$ occurs at intermediate energy, and thus as the temperature increases, the neutron spectrum shifts into the epithermal range. Figure 1 shows such a shift of the neutron spectrum into the epithermal range when changing from Hot Zero Power (HZP) to Hot Full Power (HFP) conditions.

The resonance absorption due to Doppler broadening is reflected in the calculation of the sensitivity, mainly the implicit sensitivity. This implicit sensitivity accounts for the self-shielding effect. Figure 2 illustrates the relative change in sensitivity of ${ }^{238} \mathrm{U}(n, \gamma)$ in each unit cell analyzed between HZP and HFP conditions. 
TABLE 1: Uncertainty in $k_{\text {inf }}$ in LWR unit cells.

\begin{tabular}{lcccc}
\hline Fuel & Operating conditions & $k_{\text {inf }}$ & Uncertainty in $k_{\text {inf }}(\% \Delta k / k)$ & Largest uncertainty-contributing reaction \\
\hline \multirow{2}{*}{ BWR } & HZP & 1.3382 & 0.52 & ${ }^{238} \mathrm{U}(n, \gamma)$ \\
& HFP $(40 \%$ void $)$ & 1.2208 & 0.62 & ${ }^{238} \mathrm{U}(n, \gamma)$ \\
PWR & HZP & 1.4206 & 0.48 & ${ }^{238} \mathrm{U}(n, \gamma)$ \\
& HFP & 1.4017 & 0.49 & ${ }^{238} \mathrm{U}(n, \gamma)$ \\
VVER & HZP & 1.3448 & 0.51 & ${ }^{238} \mathrm{U}(n, \gamma)$ \\
& HFP & 1.3270 & 0.52 & ${ }^{238} \mathrm{U}(n, \gamma)$ \\
KRITZ 2.1 & HZP & 1.2323 & 0.59 & ${ }^{238} \mathrm{U}(n, \gamma)$ \\
& HFP & 1.1837 & 0.63 & ${ }^{238} \mathrm{U}(n, \gamma)$ \\
KRITZ 2.13 & HZP & 1.2642 & 0.55 & ${ }^{238} \mathrm{U}(n, \gamma)$ \\
& HFP & 1.2329 & 0.58 & ${ }^{238} \mathrm{U}(n, \gamma)$ \\
\hline
\end{tabular}

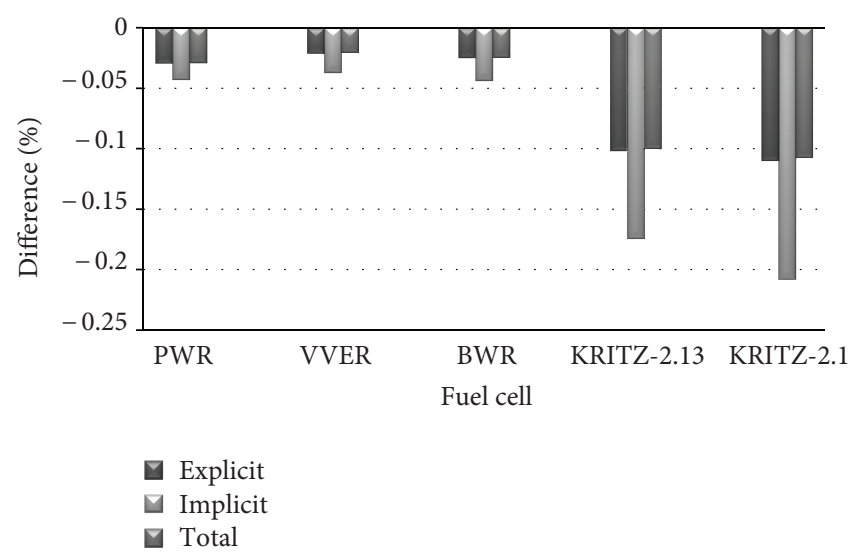

Figure 2: Relative change in the sensitivity of ${ }^{238} \mathrm{U}(n, \gamma)$.

In addition, three fuel assemblies of three light water reactor types provided by the OECD LWR UAM benchmark were analyzed. The details of the specifications are readily available [1]. The $k_{\text {inf }}$ and uncertainties obtained are shown in Table 2.

Similar to the fuel pin models, in fuel assembly models, the uncertainty in $k_{\text {inf }}$ increases with increasing temperature, while $k_{\text {inf }}$ decreases [5]. As explained earlier, this effect arises because of the increase in sensitivity of the largest contributor of uncertainty, the ${ }^{238} \mathrm{U}(n, \gamma)$ reaction. The majority of neutron capture in ${ }^{238} \mathrm{U}$ occurs at intermediate energy, and thus as the temperature increases, the spectrum shifts to the epithermal range (i.e., becomes harder). Likewise, $40 \%$ void exhibit larger uncertainty in $k_{\text {inf }}$ also due to a harder flux spectrum.

The fuel assembly analysis (as part of Exercise I-2) includes the propagation of multigroup cross-section uncertainties (multigroup covariance matrix) to twogroup homogenized cross-section uncertainties (twogroup covariance matrix). The two-group cross-section uncertainties are obtained using the SCALE-6.0 44-group covariance matrix as input to the TSUNAMI-2D sequence with GPT in SCALE 6.1. The obtained results are shown in Table 3.

One can define nine-dimensional response vector $\mathbf{R}$ $=\left[\Sigma_{a 1}, \Sigma_{a 2}, \Sigma_{f 1}, \Sigma_{f 2}, v \Sigma_{f 1}, v \Sigma_{f 2}, D_{1}, D_{2}, \Sigma_{12}\right]$ for two-group assembly homogenized cross-sections and obtain a corresponding covariance matrix in which the diagonal elements are the relative standard deviations, while off-diagonal elements are the correlation coefficients. The covariance matrices for the BWR and PWR cases are displayed in Figures 3, 4, 5 , and 6.

The obtained results for different LWR types and cases indicate the following tendencies.

(a) Group 1 (fast) cross-section uncertainty is $\sim 2-3$ times larger than Group 2 (thermal) cross-sections uncertainty.

(b) Uncertainty contributions:

(i) a major contributor to Group 1 (fast) crosssection uncertainties is U-238 inelastic scattering;

(ii) U-238 inelastic scattering uncertainty is quite large;

(iii) $40 \%$ void (and higher) exhibit larger uncertainty in $k_{\infty}$ due to harder flux spectrum.

(c) Uncertainty (correlation) contribution:

(i) U-238 inelastic scattering uncertainty is quite large and dominates correlation coefficient. 
TABLE 2: Assembly $k_{\text {inf }}$ values and associated uncertainties.

\begin{tabular}{lcccc}
\hline Fuel & Operating conditions & $k_{\text {inf }}$ & Uncertainty in $k_{\text {inf }}(\% \Delta k / k)$ & Largest uncertainty-contributing reaction \\
\hline \multirow{2}{*}{ BWR } & HZP & 1.1116 & 0.50 & ${ }^{238} \mathrm{U}(n, \gamma)$ \\
& HFP $(40 \%$ void $)$ & 1.0779 & 0.56 & ${ }^{238} \mathrm{U}(n, \gamma)$ \\
PWR & HZP & 1.4130 & 0.46 & ${ }^{23} \mathrm{U}(n, \gamma)$ \\
& HFP & 1.3968 & 0.47 & ${ }^{238} \mathrm{U}(n, \gamma)$ \\
VVER & HZP & 1.3164 & 0.47 & ${ }^{238} \mathrm{U}(n, \gamma)$ \\
& HFP & 1.3115 & 0.47 & ${ }^{238} \mathrm{U}(n, \gamma)$ \\
\hline
\end{tabular}

TABLE 3: Two-group cross-section uncertainty in LWR fuel assembly.

\begin{tabular}{|c|c|c|c|c|c|c|}
\hline \multirow{3}{*}{ Response cross-section } & \multirow{2}{*}{\multicolumn{2}{|c|}{$\begin{array}{c}\text { Uncertainty }(\% \Delta R / R) \\
\text { BWR }\end{array}$}} & \multirow{2}{*}{\multicolumn{2}{|c|}{$\begin{array}{c}\text { Uncertainty }(\% \Delta R / R) \\
\text { PWR }\end{array}$}} & \multirow{2}{*}{\multicolumn{2}{|c|}{$\begin{array}{c}\text { Uncertainty }(\% \Delta R / R) \\
\text { VVER }\end{array}$}} \\
\hline & & & & & & \\
\hline & HZP & HFP & HZP & HFP & HZP & HFP \\
\hline$\sum_{t 1}$ & 0.84 & 0.91 & 0.87 & 0.88 & 0.81 & 0.82 \\
\hline$\sum_{t 2}$ & 0.13 & 0.15 & 0.14 & 0.14 & 0.12 & 0.12 \\
\hline$D_{1}$ & 0.84 & 0.91 & 0.87 & 0.88 & 0.81 & 0.82 \\
\hline $\mathrm{D}_{2}$ & 0.13 & 0.15 & 0.14 & 0.14 & 0.12 & 0.12 \\
\hline$\sum_{a 1}$ & 0.78 & 0.83 & 0.86 & 0.87 & 0.81 & 0.82 \\
\hline$\sum_{a 2}$ & 0.20 & 0.22 & 0.22 & 0.22 & 0.21 & 0.21 \\
\hline$\sum_{f 1}$ & 0.68 & 0.72 & 0.36 & 0.36 & 0.47 & 0.47 \\
\hline$\sum_{f 2}$ & 0.32 & 0.32 & 0.32 & 0.32 & 0.32 & 0.32 \\
\hline$\sum_{11}$ & 0.84 & 0.91 & 0.87 & 0.87 & 0.81 & 0.81 \\
\hline$\sum_{12}$ & 1.10 & 1.22 & 1.20 & 1.21 & 1.03 & 1.03 \\
\hline$\sum_{21}$ & 0.27 & 0.34 & 0.30 & 0.33 & 0.26 & 0.29 \\
\hline$\sum_{22}$ & 0.13 & 0.16 & 0.15 & 0.15 & 0.13 & 0.13 \\
\hline
\end{tabular}

\begin{tabular}{|l|c|c|c|c|c|c|c|c|c|}
\hline & $\Sigma_{a 1}$ & $\Sigma_{a 2}$ & $\Sigma_{f 1}$ & $\Sigma_{f 2}$ & $v \Sigma_{f 1}$ & $\nu \Sigma_{f 2}$ & $D_{1}$ & $D_{2}$ & $\Sigma_{12}$ \\
\hline$\Sigma_{a 1}$ & 0.78 & & & & & & & & \\
\hline$\Sigma_{a 2}$ & 0.25 & 0.20 & & & & & & & \\
\hline$\Sigma_{f 1}$ & -0.47 & 0.00 & 0.68 & & & & & & \\
\hline$\Sigma_{f 2}$ & -0.04 & 0.18 & 0.13 & 0.32 & & & & & \\
\hline$\nu \Sigma_{f 1}$ & -0.43 & 0.00 & 0.87 & 0.08 & 0.98 & & & & \\
\hline$\nu \Sigma_{f 2}$ & -0.03 & 0.13 & 0.10 & 0.72 & 0.13 & 0.45 & & & \\
\hline$D_{1}$ & -0.69 & -0.02 & 0.58 & -0.02 & 0.55 & -0.02 & 0.84 & & \\
\hline$D_{2}$ & 0.03 & 0.09 & 0.01 & 0.02 & 0.00 & 0.01 & 0.04 & 0.13 & \\
\hline$\Sigma_{12}$ & 0.70 & -0.01 & -0.68 & 0.03 & -0.64 & 0.02 & -0.90 & -0.03 & 1.01 \\
\hline
\end{tabular}

FIGURE 3: BWR HZP covariance matrix.

\begin{tabular}{|l|c|c|c|c|c|c|c|c|c|}
\hline & $\Sigma_{a 1}$ & $\Sigma_{a 2}$ & $\Sigma_{f 1}$ & $\Sigma_{f 2}$ & $\nu \Sigma_{f 1}$ & $\nu \Sigma_{f 2}$ & $D_{1}$ & $D_{2}$ & $\Sigma_{12}$ \\
\hline$\Sigma_{a 1}$ & 0.83 & & & & & & & & \\
\hline$\Sigma_{a 2}$ & 0.22 & 0.22 & & & & & & & \\
\hline$\Sigma_{f 1}$ & -0.57 & 0.01 & 0.72 & & & & & & \\
\hline$\Sigma_{f 2}$ & -0.04 & 0.19 & 0.14 & 0.32 & & & & & \\
\hline$\nu \Sigma_{f 1}$ & -0.52 & 0.01 & 0.89 & 0.10 & 1.01 & & & & \\
\hline$\nu \Sigma_{f 2}$ & -0.03 & 0.14 & 0.10 & 0.72 & 0.14 & 0.45 & & & \\
\hline$D_{1}$ & -0.67 & 0.01 & 0.59 & -0.01 & 0.56 & 0.00 & 0.91 & & \\
\hline$D_{2}$ & 0.72 & -0.05 & 0.02 & 0.03 & 0.01 & 0.02 & 0.04 & 0.15 & \\
\hline$\Sigma_{12}$ & 0.03 & 0.18 & -0.72 & 0.01 & -0.68 & 0.01 & -0.84 & -0.04 & 1.22 \\
\hline
\end{tabular}

FiguRE 4: BWR HFP covariance matrix. 


\begin{tabular}{|l|c|c|c|c|c|c|c|c|c|}
\hline & $\Sigma_{a 1}$ & $\Sigma_{a 2}$ & $\Sigma_{f 1}$ & $\Sigma_{f 2}$ & $v \Sigma_{f 1}$ & $v \Sigma_{f 2}$ & $D_{1}$ & $D_{2}$ & $\Sigma_{12}$ \\
\hline$\Sigma_{a 1}$ & 0.86 & & & & & & & & \\
\hline$\Sigma_{a 2}$ & 0.13 & 0.22 & & & & & & & \\
\hline$\Sigma_{f 1}$ & 0.18 & 0.13 & 0.36 & & & & & & \\
\hline$\Sigma_{f 2}$ & 0.00 & 0.44 & 0.33 & 0.32 & & & & & \\
\hline$\nu \Sigma_{f 1}$ & -0.07 & 0.09 & 0.72 & 0.22 & 0.51 & & & & \\
\hline$\nu \Sigma_{f 2}$ & 0.00 & 0.31 & 0.24 & 0.71 & 0.33 & 0.44 & & & \\
\hline$D_{1}$ & -0.74 & 0.01 & -0.26 & 0.00 & 0.04 & 0.00 & 0.87 & & \\
\hline$D_{2}$ & 0.03 & 0.09 & 0.07 & 0.01 & 0.04 & 0.01 & 0.05 & 0.14 & \\
\hline$\Sigma_{12}$ & 0.76 & -0.04 & 0.25 & 0.00 & -0.07 & 0.00 & -0.89 & -0.04 & 1.20 \\
\hline
\end{tabular}

Figure 5: TMI HZP covariance matrix.

\begin{tabular}{|l|c|c|c|c|c|c|c|c|c|}
\hline & $\Sigma_{a 1}$ & $\Sigma_{a 2}$ & $\Sigma_{f 1}$ & $\Sigma_{f 2}$ & $\nu \Sigma_{f 1}$ & $\nu \Sigma_{f 2}$ & $D_{1}$ & $D_{2}$ & $\Sigma_{12}$ \\
\hline$\Sigma_{a 1}$ & 0.87 & & & & & & & & \\
\hline$\Sigma_{a 2}$ & 0.13 & 0.22 & & & & & & & \\
\hline$\Sigma_{f 1}$ & 0.17 & 0.13 & 0.36 & & & & & & \\
\hline$\Sigma_{f 2}$ & 0.00 & 0.44 & 0.33 & 0.32 & & & & & \\
\hline$\nu \Sigma_{f 1}$ & -0.08 & 0.09 & 0.72 & 0.22 & 0.51 & & & & \\
\hline$\nu \Sigma_{f 2}$ & 0.00 & 0.32 & 0.24 & 0.71 & 0.33 & 0.44 & & & \\
\hline$D_{1}$ & -0.74 & 0.01 & -0.26 & 0.00 & 0.04 & 0.00 & 0.88 & & \\
\hline$D_{2}$ & 0.03 & 0.10 & 0.06 & 0.01 & 0.04 & 0.01 & 0.05 & 0.14 & \\
\hline$\Sigma_{12}$ & 0.77 & -0.04 & 0.25 & 0.00 & -0.07 & 0.00 & -0.89 & -0.04 & 1.21 \\
\hline
\end{tabular}

FIGURE 6: TMI HFP covariance matrix.

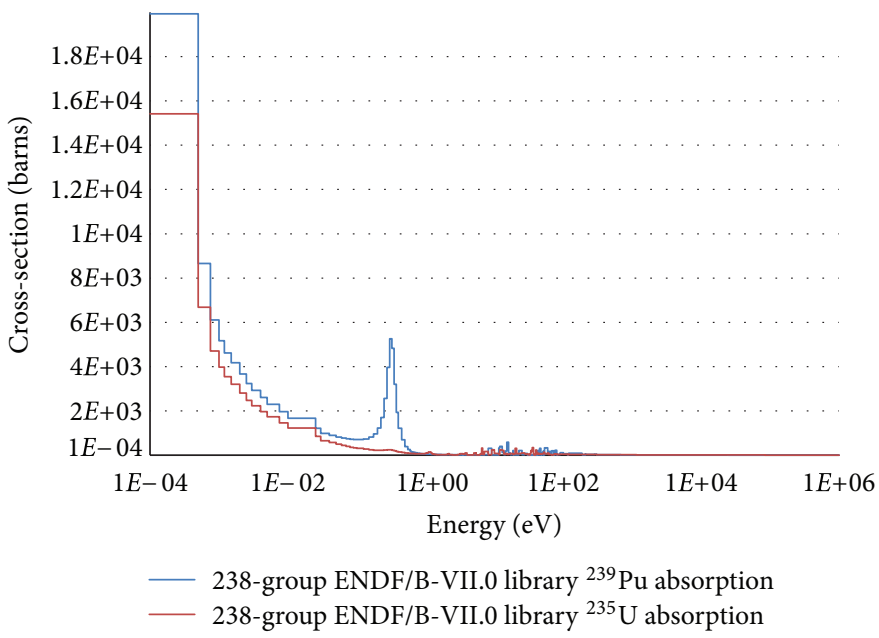

Figure 7: Plot of ${ }^{239} \mathrm{Pu}$ absorption cross-sections compared to ${ }^{235} \mathrm{U}$ absorption cross-sections.

TABLE 4: Uncertainty in $k_{\text {inf }}$ in GEN-III unit cells.

\begin{tabular}{lcccc}
\hline Fuel & Compositions & $k_{\text {inf }}$ & Uncertainty in $k_{\text {inf }}(\% \Delta k / k)$ & Largest uncertainty-contributing reaction \\
\hline \multirow{3}{*}{ MOX } & $9.8 \%{ }^{239} \mathrm{Pu}$ & 1.0921 & 0.94 & ${ }^{238} \mathrm{U}\left(n, n^{\prime}\right)$ \\
& $6.5 \%{ }^{239} \mathrm{Pu}$ & 1.0540 & 0.97 & ${ }^{238} \mathrm{U}\left(n, n^{\prime}\right)$ \\
\hline \multirow{2}{*}{$\mathrm{UOX}$} & $3.7 \%{ }^{239} \mathrm{Pu}$ & 1.0115 & 0.99 & ${ }^{238} \mathrm{U}\left(n, n^{\prime}\right)$ \\
& $4.2 \%{ }^{235} \mathrm{U}$ & 1.2431 & 0.51 & ${ }^{238} \mathrm{U}(n, \gamma)$ \\
& $3.2 \%{ }^{235} \mathrm{U}$ & 1.1741 & 0.54 & ${ }^{238} \mathrm{U}(n, \gamma)$ \\
\hline \multirow{2}{*}{$\mathrm{UOX}-\mathrm{Gd}_{2} \mathrm{O}_{3}$} & $2.1 \%{ }^{235} \mathrm{U}$ & 1.0490 & 0.59 & ${ }^{238} \mathrm{U}(n, \gamma)$ \\
\hline
\end{tabular}




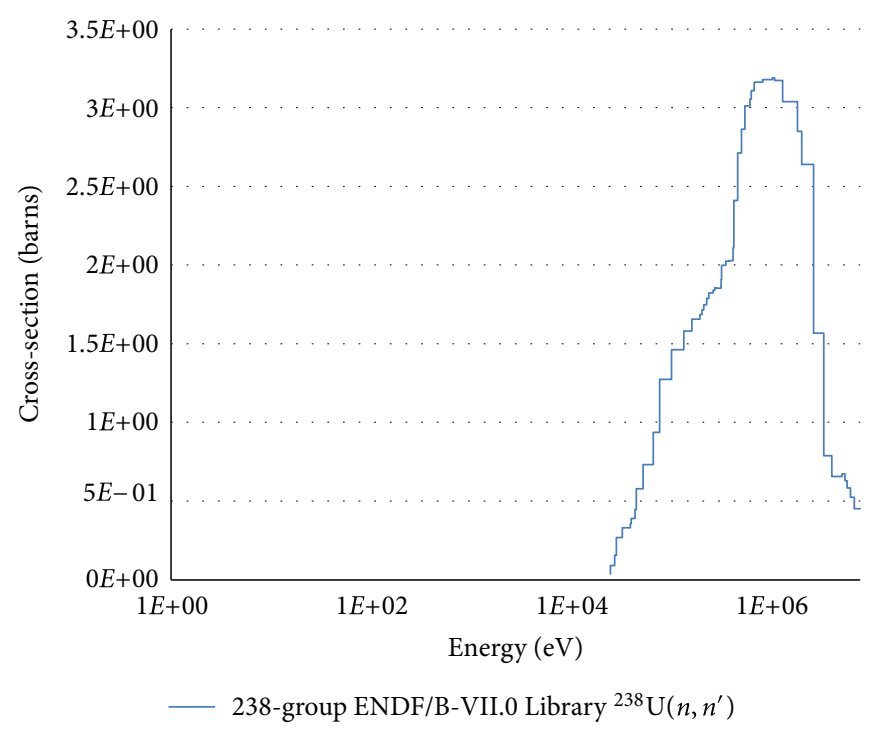

FIgURE 8: The plot of inelastic cross-section of ${ }^{238} \mathrm{U}$ as a function of energy.

TABLE 5: Macroscopic fission cross-sections in MOX fuel cells.

\begin{tabular}{lcccc}
\hline Fuel & Composition & $k_{\text {inf }}$ & ${ }^{235} \mathrm{U} \sum_{f}(1 / \mathrm{cm})$ & ${ }^{239} \mathrm{Pu} \sum_{f}(1 / \mathrm{cm})$ \\
\hline MOX & $3.7 \%$ Pu329 & 1.0115 & 6.70 & 13.74 \\
MOX & $6.5 \%$ Pu329 & 1.0540 & 4.65 & 8.40 \\
MOX & $9.8 \%$ Pu329 & 1.0921 & 3.69 & 5.88 \\
\hline
\end{tabular}

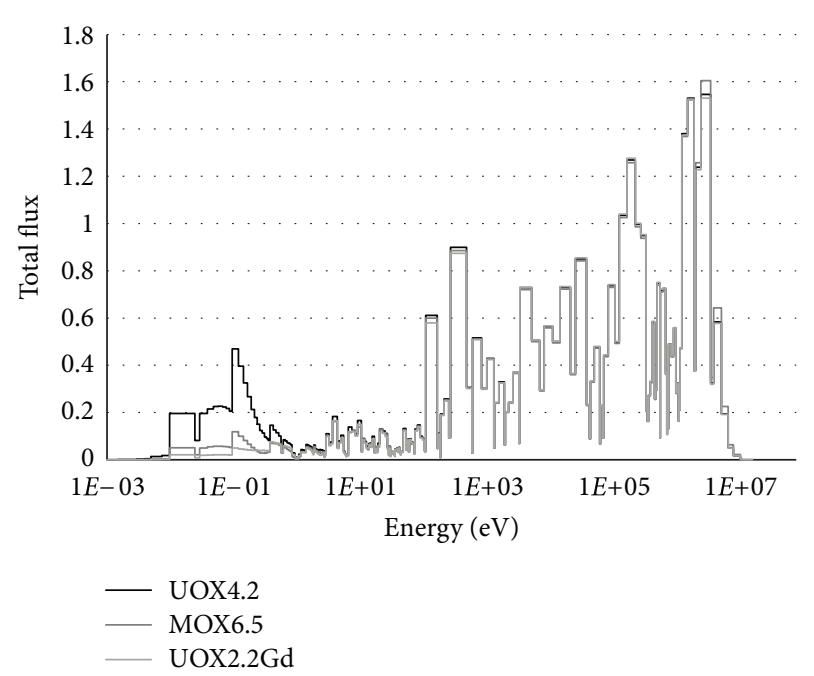

FIGURE 9: The neutron flux spectra of three unit cell compositions.

TABLE 6: LWR neutronics parameters [6, Table 3-1 page 88].

\begin{tabular}{lcc}
\hline Parameter & ${ }^{235} \mathrm{U}$ & ${ }^{239} \mathrm{Pu}$ \\
\hline Average $v$ & 2.4 & 2.9 \\
Average $\eta$ & 2.0 & 1.9 \\
Average $\sigma_{\text {fission }}$ & 280 barns & 790 barns \\
\hline
\end{tabular}

\section{Composition Sensitivity Study}

Selected fuel pin cells and four types of fuel assemblies from a representative Generation III LWR (GEN-III) specification were analyzed for the purpose of comparing effect of the compositions on the uncertainty calculations. The specifications of the GEN-III unit cells and fuel assemblies are readily available [1].

Three types of unit cells were analyzed at Hot Full Power; these include MOX, UOX, and UOX with $\mathrm{Gd}_{2} \mathrm{O}_{3}$. The multiplication factors and their uncertainties are presented in Table 4.

For each group of the fuel cells, several factors influence the changes in the uncertainty in $k_{\text {inf }}$, and each will be examined separately.

For each unit cell, the calculated $k_{\text {inf }}$ increases with increasing enrichment of fissile material, while the uncertainty in $k_{\text {inf }}$ decreases.

For the MOX fuel cells, the amount of ${ }^{238} \mathrm{U}$ is reduced as the amount of ${ }^{239} \mathrm{Pu}$, the fissile material, is increased. The reduction in the amount of ${ }^{238} \mathrm{U}$ means that there is less neutrons absorption by ${ }^{238} \mathrm{U}$ nuclides. This is later found to be the most important nuclide contributor to uncertainty in $k_{\text {inf }}$ in the unit cell. Thus, with less absorption by ${ }^{238} \mathrm{U}$ nuclides, the uncertainty is reduced when comparing only the changes in the enrichment of ${ }^{239} \mathrm{Pu}$ in MOX fuel cell. 


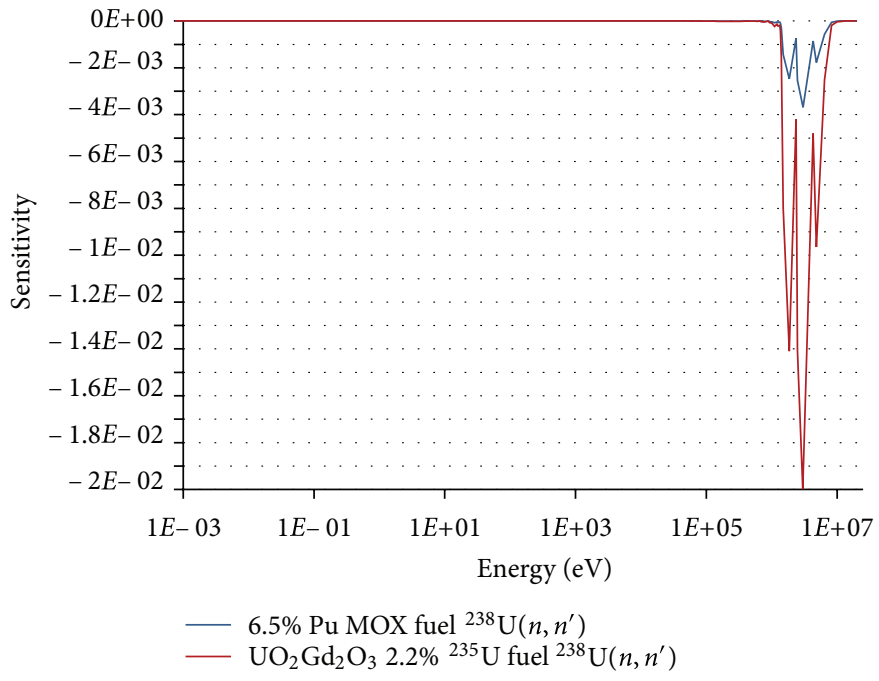

FIgURE 10: The sensitivity of ${ }^{238} \mathrm{U}\left(n, n^{\prime}\right)$ as function of energy.

TABLE 7: Uncertainty in $k_{\text {inf }}$ in GEN-III fuel assembly.

\begin{tabular}{lcccc}
\hline Fuel & Compositions & $k_{\text {inf }}$ & Uncertainty in $k_{\text {inf }}(\% \Delta k / k)$ & Largest uncertainty-contributing reaction \\
\hline \multirow{3}{*}{ GEN III } & Type 1 & 1.2501 & 0.49 & ${ }^{238} \mathrm{U}(n, \gamma)$ \\
& Type 2 & 1.1228 & 0.49 & ${ }^{238} \mathrm{U}(n, \gamma)$ \\
& Type 3 & 0.9564 & 0.53 & ${ }^{238} \mathrm{U}(n, \gamma)$ \\
& Type 4 & 1.0700 & 0.97 & ${ }^{238} \mathrm{U}\left(n, n^{\prime}\right)$ \\
\hline
\end{tabular}

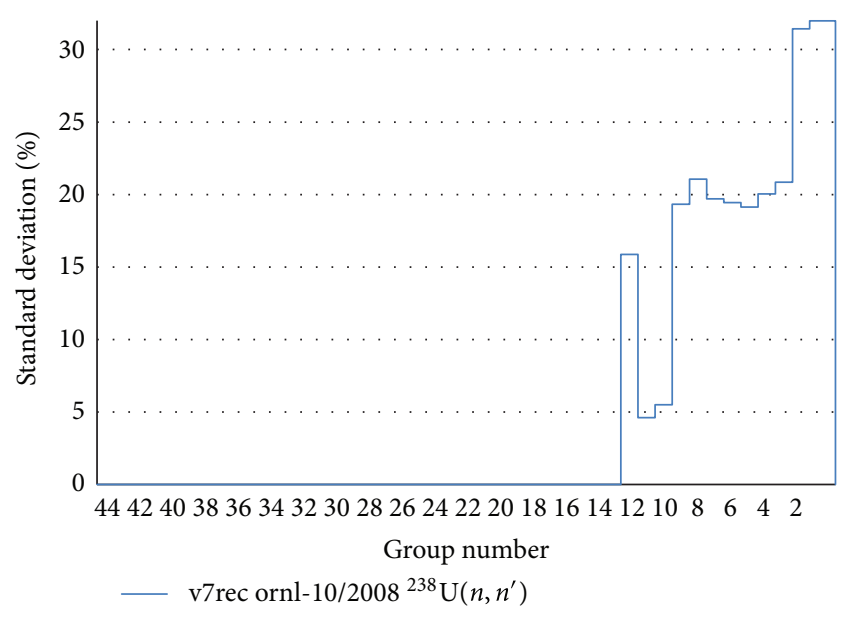

Figure 11: The 2D plot of ${ }^{238} \mathrm{U}\left(n, n^{\prime}\right)$ covariance by energy group.

However, the uncertainties of the MOX fuel cells were nearly twice than that of UOX fuel. The presence of ${ }^{239} \mathrm{Pu}$ plays an important role in the increase in uncertainty. Figure 7 shows that the neutron absorption by ${ }^{239} \mathrm{Pu}$ outcompeted the neutron absorption by ${ }^{235} \mathrm{U}$.

As a consequence, from the fact that more neutrons are absorbed by ${ }^{239} \mathrm{Pu}$ than ${ }^{235} \mathrm{U}$, more neutrons are produced by fission due to ${ }^{239} \mathrm{Pu}$. Table 5 shows the dependency of fission macroscopic cross-sections to the composition of the

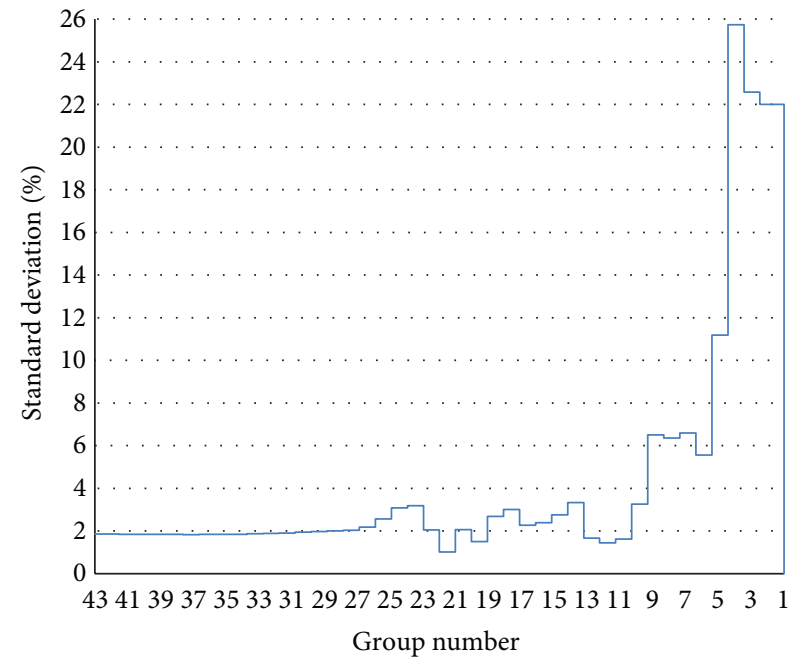

— v7rec ornl- $10 / 2008^{238} \mathrm{U}(n, \gamma)$

Figure 12: The 2D plot of ${ }^{238} \mathrm{U}(n, \gamma)$ covariance by energy group.

fuel, and Table 6 displays the comparison of the neutrons production parameters of each nuclide.

Neutrons produced by ${ }^{239} \mathrm{Pu}$, in general, will have higher energy than that of ${ }^{235} \mathrm{U}$. In this case, the neutron spectrum is harder because more neutrons with higher energies are produced by ${ }^{239} \mathrm{Pu}$, the dominant fission nuclide. 
TABLE 8: Two-group cross-section uncertainty $(\% \Delta R / R)$ in GEN-III fuel assembly.

\begin{tabular}{|c|c|c|c|c|}
\hline Response cross-section & GEN III type 1 & GEN III type 2 & GEN III type 3 & GEN III type 4 \\
\hline$\overline{\sum_{t 1}}$ & 0.90 & 0.90 & 0.90 & 0.97 \\
\hline$\sum_{t 2}$ & 0.14 & 0.14 & 0.14 & 0.14 \\
\hline$D_{1}$ & 0.90 & 0.90 & 0.90 & 0.97 \\
\hline$D_{2}$ & 0.14 & 0.14 & 0.14 & 0.14 \\
\hline$\sum_{a 1}$ & 0.89 & 0.89 & 0.89 & 1.00 \\
\hline$\sum_{a 2}$ & 0.21 & 0.19 & 0.19 & 0.24 \\
\hline$\sum_{f 1}$ & 0.37 & 0.37 & 0.50 & 0.44 \\
\hline$\sum_{f 2}$ & 0.32 & 0.32 & 0.33 & 0.62 \\
\hline$\sum_{11}$ & 0.90 & 0.90 & 0.90 & 0.97 \\
\hline$\sum_{12}$ & 1.25 & 1.25 & 1.24 & 1.47 \\
\hline$\sum_{21}$ & 0.33 & 0.32 & 0.31 & 0.37 \\
\hline$\sum_{22}$ & 0.15 & 0.15 & 0.15 & 0.16 \\
\hline
\end{tabular}

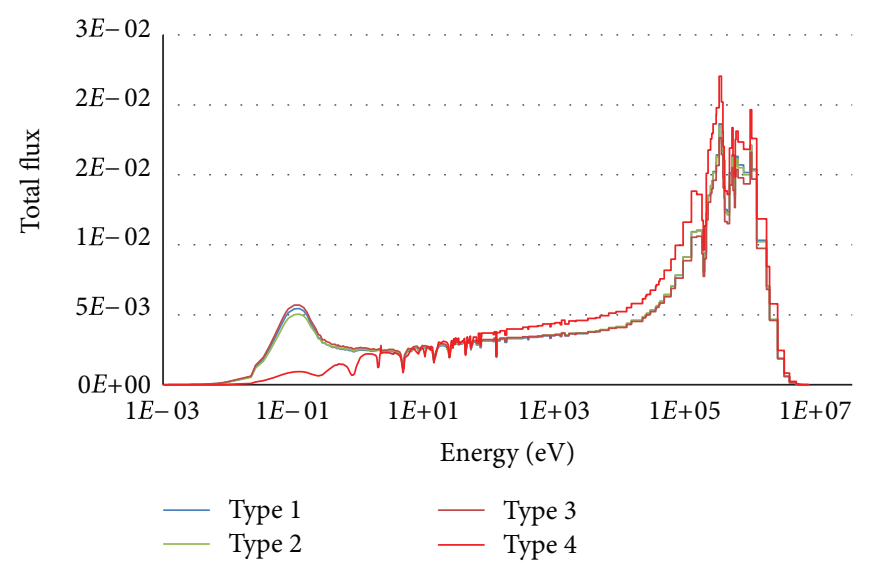

FIgURE 13: The neutron flux spectra of GEN-III LWR fuel assembly.

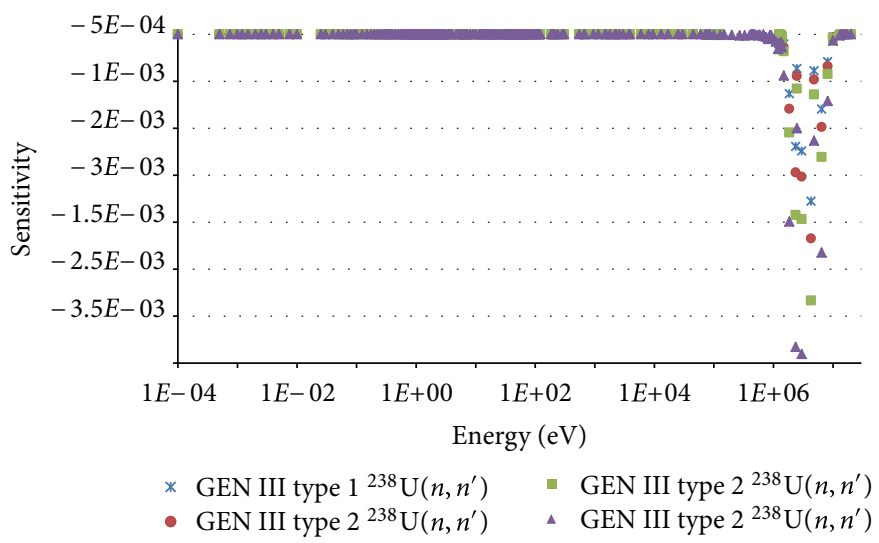

FIGURE 14: The sensitivity of ${ }^{238} \mathrm{U}\left(n, n^{\prime}\right)$ as function of energy. 
On the other hand, for UOX fuel cell with $\mathrm{Gd}_{2} \mathrm{O}_{3}$ added, similar finding (hardening of the neutron spectrum) occurred but due to a very different mechanism. The uncertainties in $k_{\text {inf }}$ are nearly triple of the fuel cell with similar ${ }^{235} \mathrm{U}$ enrichment. This is due to the fact that gadolinium is a major thermal neutron absorber. The significant reduction in the thermal neutrons shifted the fission reactions toward neutrons with higher energy. Once again, the neutron spectrum is harder than that of UOX fuel cell without $\mathrm{Gd}_{2} \mathrm{O}_{3}$ added.

The uncertainties in fuel cells with harder neutron spectrum seemed to be higher than fuel cells with softer neutron spectrum. This is due to the absorption of the ${ }^{238} \mathrm{U}$. The resonance absorption of ${ }^{238} \mathrm{U}$ that occurs at higher neutron energy is very large.

The absorption of thermal neutrons by ${ }^{239} \mathrm{Pu}$ dominates the fission process. Since fission in ${ }^{239} \mathrm{Pu}$ produces more fast neutrons, the neutron spectrum becomes harder. The harder the spectrum, the higher the ${ }^{238} \mathrm{U}\left(n, n^{\prime}\right)$ reaction rates. This effect can be seen in Figure 8, which depicts the cross-section of ${ }^{238} \mathrm{U}\left(n, n^{\prime}\right)$ reaction. When $\mathrm{Gd}_{2} \mathrm{O}_{3}$ is added, the largest nuclide reaction cross-section contributor to the uncertainty in $k_{\text {inf }}$ changed from ${ }^{238} \mathrm{U}(n, \gamma)$ to ${ }^{238} \mathrm{U}\left(n, n^{\prime}\right)$. These changes are due to the presence of gadolinium, mainly a thermal neutron absorber, causing significant reductions in thermal neutron populations. The neutron flux spectrum is harder, and it can be seen in Figure 9.

The sensitivity profiles were compared in Figure 10. It was found that the unit cell containing gadolinium is very sensitive to the ${ }^{238} \mathrm{U}\left(n, n^{\prime}\right)$ reaction, and this large sensitivity contributes to increase in uncertainty.

The covariance matrix of ${ }^{238} \mathrm{U}\left(n, n^{\prime}\right)$ is presented in Figure 11, while the covariance matrix of ${ }^{238} \mathrm{U}(n, \gamma)$ is shown in Figure 12. It should be noted that at high energy, the coefficients of relative covariance of ${ }^{238} \mathrm{U}\left(n, n^{\prime}\right)$ cross-section reach $30 \%$ of the standard deviation. Thus, for a reactor with a harder spectrum, it produces a large contribution to the uncertainty of $k_{\text {inf }}$.

Four types of fuel assembly from a representative Generation III LWR specification (as part of Exercise I-2) were analyzed at Hot Full Power condition:

(i) type 1 (UOX $4.2 \%{ }^{235} \mathrm{U}$ );

(ii) type 2 (UOX $4.2 \%{ }^{235} \mathrm{U}+\mathrm{UO}_{2} \mathrm{Gd}_{2} \mathrm{O}_{3} 2.2 \%{ }^{235} \mathrm{U}$ );

(iii) type 3 (UOX $3.2 \%{ }^{235} \mathrm{U}+\mathrm{UO}_{2} \mathrm{Gd}_{2} \mathrm{O}_{3} 1.9 \%{ }^{235} \mathrm{U}$ );

(iv) type 4 (MOX).

The details of the specifications are readily available [1]. The values calculated for $k_{\text {inf }}$ and their uncertainties are presented in Table 7.

Results show that $k_{\text {inf }}$ increases with increasing enrichment of fissile material, while the uncertainty in $k_{\text {inf }}$ decreases. Unlike the effect we found earlier for UOX fuel pin cell with $\mathrm{Gd}_{2} \mathrm{O}_{3}$ added, in this case the presence of $\mathrm{Gd}_{2} \mathrm{O}_{3}$ does not lead to a significant increase in uncertainty because of the homogenization process. For the MOX fuel assembly, there is a remarkable increase in uncertainty in $k_{\text {inf }}$, and this result is similar to that observed in the corresponding pin cell analysis.

Additionally, the two-group cross-section uncertainties are presented in Table 8 .

These results show, as expected, a larger uncertainty for the MOX fuel assembly (type 4) than for the UOX assemblies (types 1 through 3 ).

Figure 13 depicts the neutron flux of fuel assemblies from a representative GEN-III LWR at HFP condition. There is a significant reduction in the thermal neutrons for the MOX fuel assembly. The harder the spectrum, the higher the ${ }^{238} \mathrm{U}\left(n, n^{\prime}\right)$ reaction rates.

Sensitivity profiles of ${ }^{238} \mathrm{U}\left(n, n^{\prime}\right)$ were compared in Figure 14. Fuel assembly containing $\mathrm{Pu}$ is very sensitive to ${ }^{238} \mathrm{U}\left(n, n^{\prime}\right)$ reaction, and its contribution to uncertainty is noticeable.

\section{Conclusions}

Sensitivity studies have been performed using SCALE [7] to investigate the effect of temperature and material composition on cross-section uncertainty propagation at two levelspin cell models (within the framework of Exercise I-1) and assembly models (within the framework of Exercise I-2). It was found that the uncertainty in $k_{\text {inf }}$ is dominated by neutron interactions with $238 \mathrm{U}$ in both studies with varying temperatures and compositions. The following tendencies have been observed:

(a) increasing temperature leads to increasing uncertainty in $k_{\text {inf }}$;

(b) decreasing ${ }^{238} \mathrm{U}$ in fuel composition leads to decreasing uncertainty in $k_{\text {inf }}$;

(c) major contributor to uncertainty is affected by the neutron spectrum.

The fast group cross-section uncertainties are much larger than the thermal cross-section uncertainties due to the larger role of ${ }^{238} \mathrm{U}$.

\section{Future Work}

The future studies will be focused on Exercise I-3propagation of few-group cross-section uncertainties to the core steady state stand-alone neutronics calculations using statistical methodology similar to the one reported in [8].

\section{Acknowledgments}

The authors would like to acknowledge the two Ph.D. fellowship grants, one at the Technical University of Catalonia (sponsored by the Spanish Nuclear Security Council) and the other at the Pennsylvania State University (sponsored by the US Nuclear Regulatory Commission), which supported the graduate students performing the studies reported in this paper. 


\section{References}

[1] "OECD/NEA benchmark for Uncertainty Analysis in Modelling (UAM) for design, operation and safety analysis of LWRs," in Specification and Support Data For the Neutronics Cases (Phase I), vol. 1, Version 2. 0. NEA/NSC/DOC(2012), 2012.

[2] D. Cacuci, Sensitivity and Uncertainty Analysis: Theory, vol. 1, Chapman \& Hall/CRC, 2003.

[3] "SCALE: a comprehensive modeling and simulation suite for nuclear safety analysis and design," ORNL/TM-2005/39, Version 6. 1, Oak Ridge National Laboratory, Oak Ridge, Tenn, USA, 2011.

[4] S. Kamerow, C. Arenas Moreno, and K. Ivanov, "Uncertainty analysis of light water reactor unit fuel pin cells," in Proceedings of the International Conference on Mathematics and Computational Methods Applied to Nuclear Science and Engineering (MઐC '11), Rio de Janeiro, Brazil, May 2011.

[5] M. Avramova, C. Arenas Moreno, and K. Ivanov, "Extension of BEPU methods to sub-channel thermal-hydraulics and to coupled three-dimensional neutronics/thermal-hydraulics codes," in Proceedings of The OECD/CSNI Workshop, Barcelona, Spain, November 2011.

[6] J. Duderstadt and L. Hamilton, Nuclear Reactor Analysis, John Wiley \& Sons, New York, NY, USA, 1976.

[7] B. T. Rearden, M. Williams, M. Jessee, D. Mueller, and D. Wiarda, "Sensitivity and uncertainty analysis capabilities and data in SCALE," Nuclear Technology, vol. 174, no. 2, pp. 236-288, 2011.

[8] M. Klein, L. Gallner, B. Krzykacz-Hausmann, A. Pautz, and W. Zwermann, "Influence of nuclear data uncertainties on reactor core calculations," Kerntechnik, vol. 76, no. 3, pp. 174-178, 2011. 


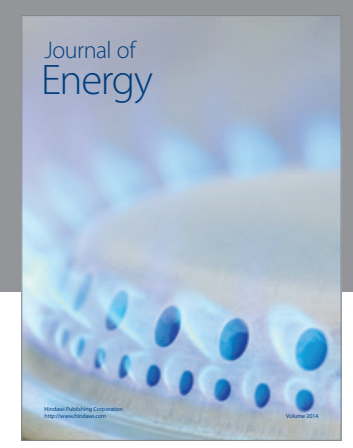

Journal of

Industrial Engineering
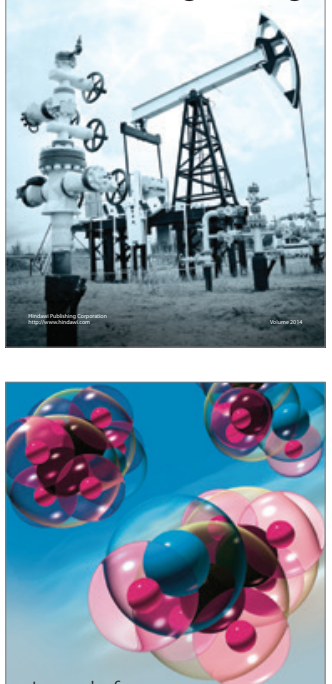

Fuels
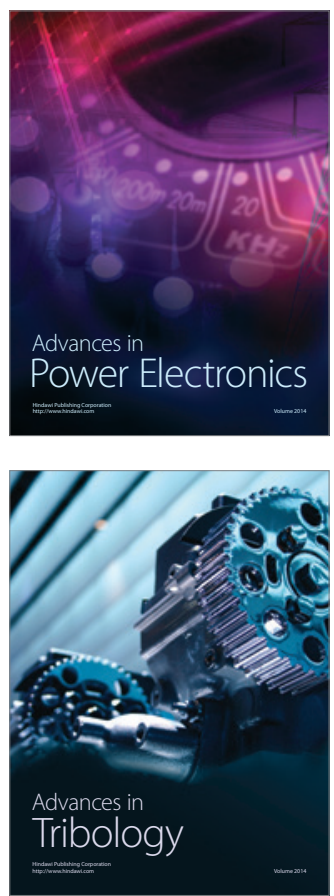

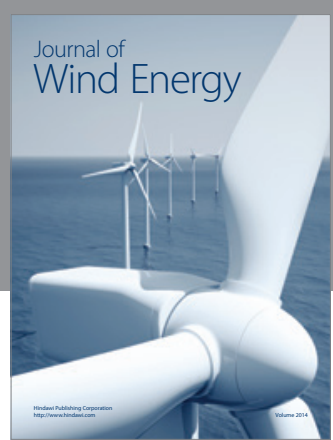

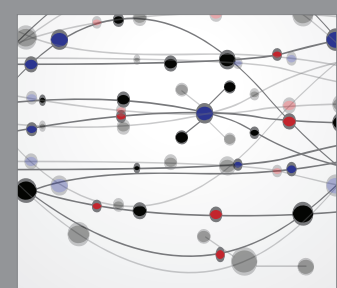

The Scientific World Journal

Submit your manuscripts at http://www.hindawi.com

Journal of

Structures
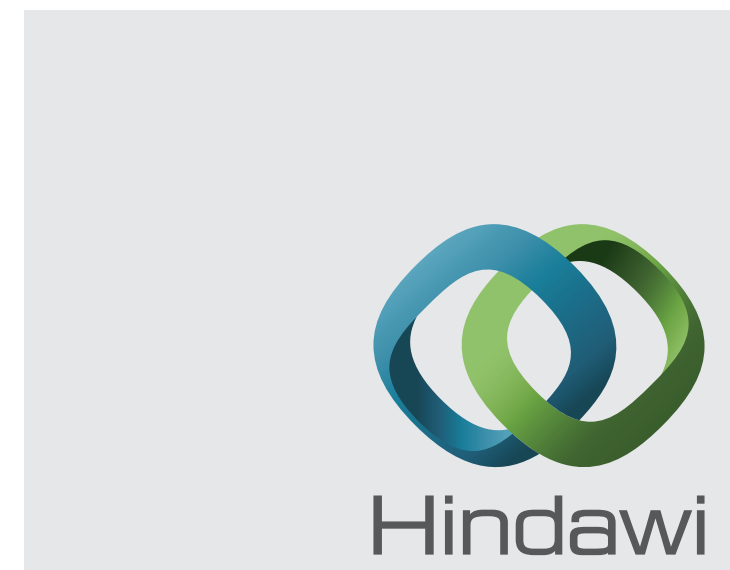

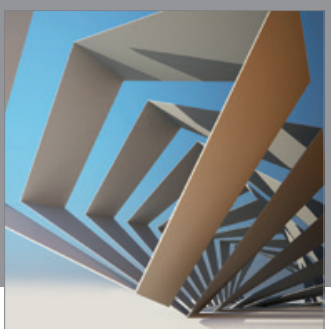

Rotating

Machinery
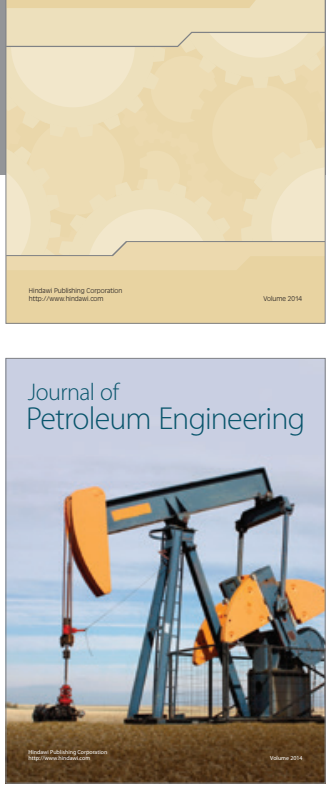

Journal of

Solar Energy
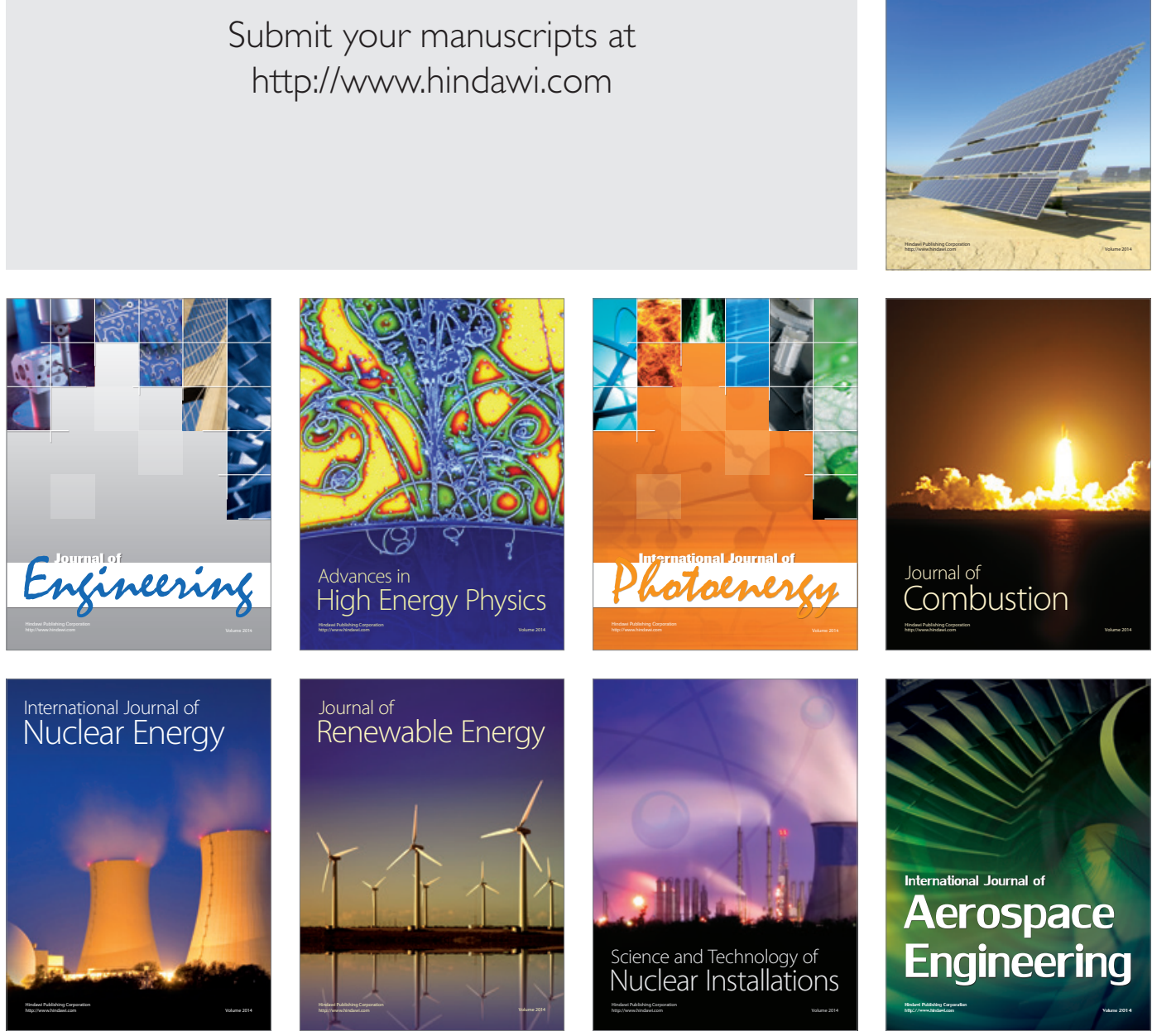